Ann. Biol. anim. Bioch. Biophys., 1979, 19 (2 A), 367-373.

\title{
Essai de conservation prolongée de gamètes à basse température, sans congélation
}

\author{
par L. HENRIET, P. ESTERMANS, B. CUCHE, J. H. MAISON
}

Université Cotholique de Louvoin et Instituf Belge d'Etude des Hautes Pressions. Sciences Vétérinaires

3, place de la Croix du Sud 1348 Louvain-la-Neuve, Belgique.

Summary. Trial of long-term gamete storage af low temperature without freezing.

In this study we attempted long-term storage of living cells (particularly gametes) at a temperature lower than $0{ }^{\circ} \mathrm{C}$. Contrary to other methods, avoiding cell disorganization no anti-crystallization agent was used. Preservation was obtained by lowering the freezing point of the cell medium by increasing the pressure. The cells thus treated emerged from the tests apparently intact, but the spermatozoa were not motile.

\section{Introduction.}

Nous avons cherché à conserver des gamètes et de jeunes embryons, pendant une longue période, en évitant les inconvénients inhérents à la congélation des cellules vivantes : le bouleversement des structures cellulaires par les cristaux de glace oblige à recourir à un cryoprotecteur qui peut modifier l'activité des cellules avant et après congélation. Le principe de la méthode consiste à prévenir la cristallisation de l'eau intracellulaire en combinant un abaissement de température sous zéro degré à un accroissement de pression. En effet, de nombreux travaux (cf. bibliographie) ont eu pour objet la résistance à de hautes pressions de bactéries, de virus ou encore de constituants cellulaires et leurs résultats ont montré que ce materiel peut résister à des pressions supérieures à la normale.

\section{Matériel et méthodes.}

a) Les cellules conservées.

Trois types de cellules ont été enfermées simultanément dans la chambre à pression :

- du sperme préparé pour l'insémination ;

- des ovocytes prélevés dans leur fluide folliculaire et provenant aussi bien de petits follicules que de follicules proches de l'ovulation ;

- du sang citraté. 
Le sperme pur subit l'effet de son propre autoantibiotisme et l'acide lactique qu'il produit entraîne rapidement un blocage du mouvement (Devuyst et Henriet, 1960). Le temps requis pour la mise en condition de l'appareillage nous a donc obligé à employer du sperme préparé pour l'insémination artificielle.

Nous avons observé que l'ovocyte mûr résiste moins bien que l'ovocyte immature au cours de manipulations in vitro et nous avons cherché à mettre en évidence une différence éventuelle de comportement à l'égard des augmentations de pression.

Quant aux cellules sanguines, elles ont été introduites dans nos essais d'abord à titre de matériel de comparaison et en outre, en raison de l'intérêt que pourrait susciter un tel moyen de conservation.

\section{b) Les récipients de conservation.}

Différents récipients à parois déformables ont été utilisés de manière à assurer la transmission des pressions. Parmi les paillettes d'insémination de Cassou, les seringues munies de leur piston, les poires en caoutchouc et les tubes métalliques à pommade, ce sont ces derniers qui nous ont paru les plus maniables.

c) Les étapes de refroidissement ef les variations de pression.

Les cellules doivent être mises sous pression avant de passer sous zéro degré et l'appareillage doit être refroidi ensuite. Malheureusement, comme la chambre de compression représente un gros volant calorique, nous avons dû procéder à un premier refroidissement jusqu'à 1 degré, de manière à réduire rapidement les activités enzymatiques. La seconde étape de refroidissement se place immédiatement après l'obtention de la pression nécessaire. Nous avons refroidi jusqu'à $-15^{\circ} \mathrm{C}$ sous pression de $1600 \mathrm{~kg} / \mathrm{cm}^{2}$. Ces limites ont été imposées par l'appareillage utilisé. Lors de l'arrêt de conservation, il faut refaire le même chemin en sens inverse : la pression doit être maintenue jusqu'au retour au-dessus de zéro degré. Le refroidissement jusqu'à 1 degré exige $3 \mathrm{~h}$ pour le sperme, il en a été de même pour les ovocytes ef le sang. Les $800 \mathrm{~kg}$ sont atteints en $5 \mathrm{mn}$. La chambre de pression est introduite dans l'armoire à $-15^{\circ} \mathrm{C}$ et les $1600 \mathrm{~kg}$ sont atteints en $10 \mathrm{mn}$. Le réchauffage a été opéré à température du laboratoire et la décompression a duré respectivement 5,10 et $20 \mathrm{mn}$. Les temps de conservation ont été de 5,6 et 10 jours.

\section{Résultats.}

Le résultat le plus encourageant est une restitution des cellules dans leur état normal. Si on en juge d'après leur image sous le microscope à contraste de phase, aucune altération ne s'est manifestée et les frottis de sang, colorés par les méthodes usuelles, semblent normaux. Par contre, les spermatozoïdes sont ressortis immobiles ef incapables de décolorer une solution de bleu de méthylène. Leur survie n'a pas été assurée et pour autant qu'elle puisse servir de témoin, elle nous fait douter de la survie des autres cellules. 


\section{Discussion.}

La force de cohésion des atomes s'accroît fortement lorsque la température s'abaisse. En effet, le diagramme qui lie les pressions et les températures de cristallisation montre que pour chaque abaissement de 1 degré, sous le zéro de l'échelle centigrade, on doit exercer une pression de $100 \mathrm{~kg}$ pour maintenir l'état liquide (fig. 1).

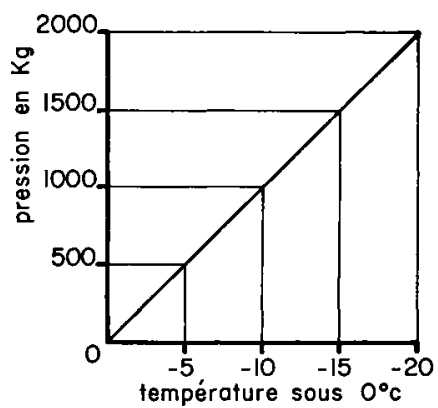

FIG. 1. - Diagramme des pressions ef des températures de cristallisation.

On conclura facilement que pareille technique réclame des appareillages dont la résistance augmente avec les pression recherchées. Nous disposions d'un appareil permettant d'atteindre des pressions de $2000 \mathrm{~kg}$ (fig. 2). La température de conservation ne pouvait donc pas descendre au-delà de $20^{\circ} \mathrm{C}$ sous zéro et pour garder une marge de sécurité, nous n'avons pas dépassé la zone des $-15^{\circ} \mathrm{C}$. Toutefois, nous ne pouvons pas certifier que cefte température constitue une condition idéale de longue conservation. En effet, lorsqu'on congèle, on place les organites et les molécules dans l'impossibilité d'agir et en principe, dès qu'on passe au-dessous de zéro, la vie est suspendue.

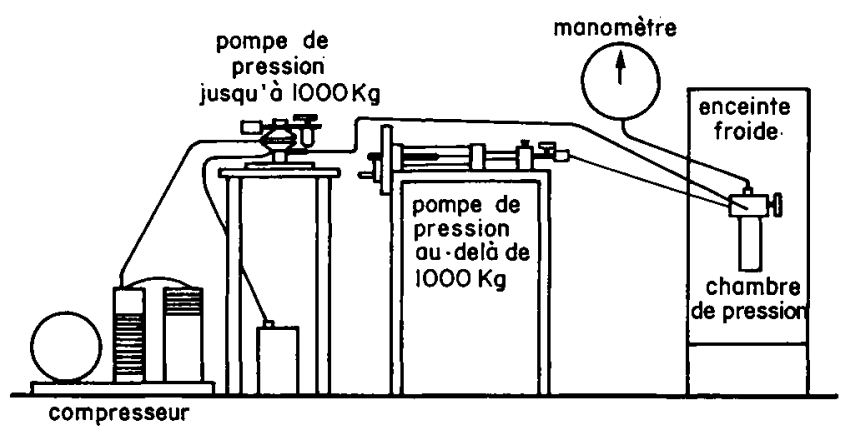

FIG. 2. - Appareillages utilisés (schéma).

Lorsque le milieu reste fluide, rien n'est certain. Tout ce dont on peut présumer, c'est que les enzymes sont inactives mais encore ne peut-on écarter l'hypothèse d'une distorsion dans l'activité. 
Il y aurait donc intérêt à atteindre des températures plus basses mais cela ne se peut qu'au prix de pressions énormes. Or, les $6000 \mathrm{~kg}$ nécessaires pour descendre jusqu'aux environs de 600, altèrent les globules rouges (Dow et Matthews, 1939). Il y a donc une limite que nous nous occupons à définir.

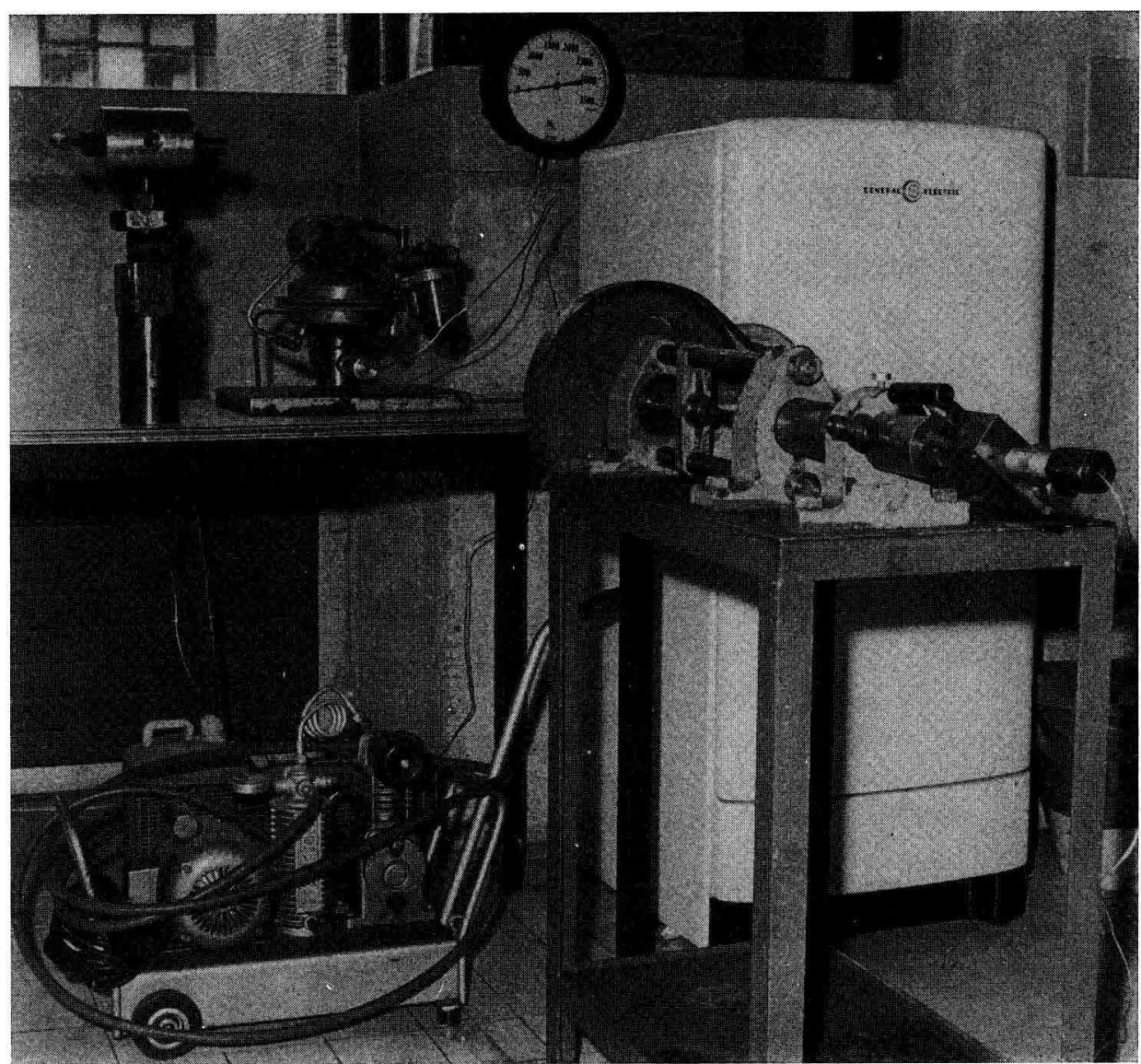

PHOTO 1. - Apporeillage de haute pression.

Nous constatons que, pour le sperme, le milieu de conservation est convenable tandis que nous devons envisager des supports d'énergie pour l'ovocyte qui doit surm vivre jusqu'à l'abaissement de température.

On peut aussi envisager de compléter le blocage enzymatique par une action autoantibiotique ou par un changement de $\mathrm{pH}$.

PHOTO 2. - Spermatozoides photographiés après 6 jours de conservation.

PHOTO 3. - Spermatozoïdes photographiés après 10 jours de conservation (coloration au Wasserblau/Rose de Bengale).

PHOTOS 4, 5. - Ovocyles recueillis après 6 jours ef 10 jours de conservation (contraste interférentiel) 

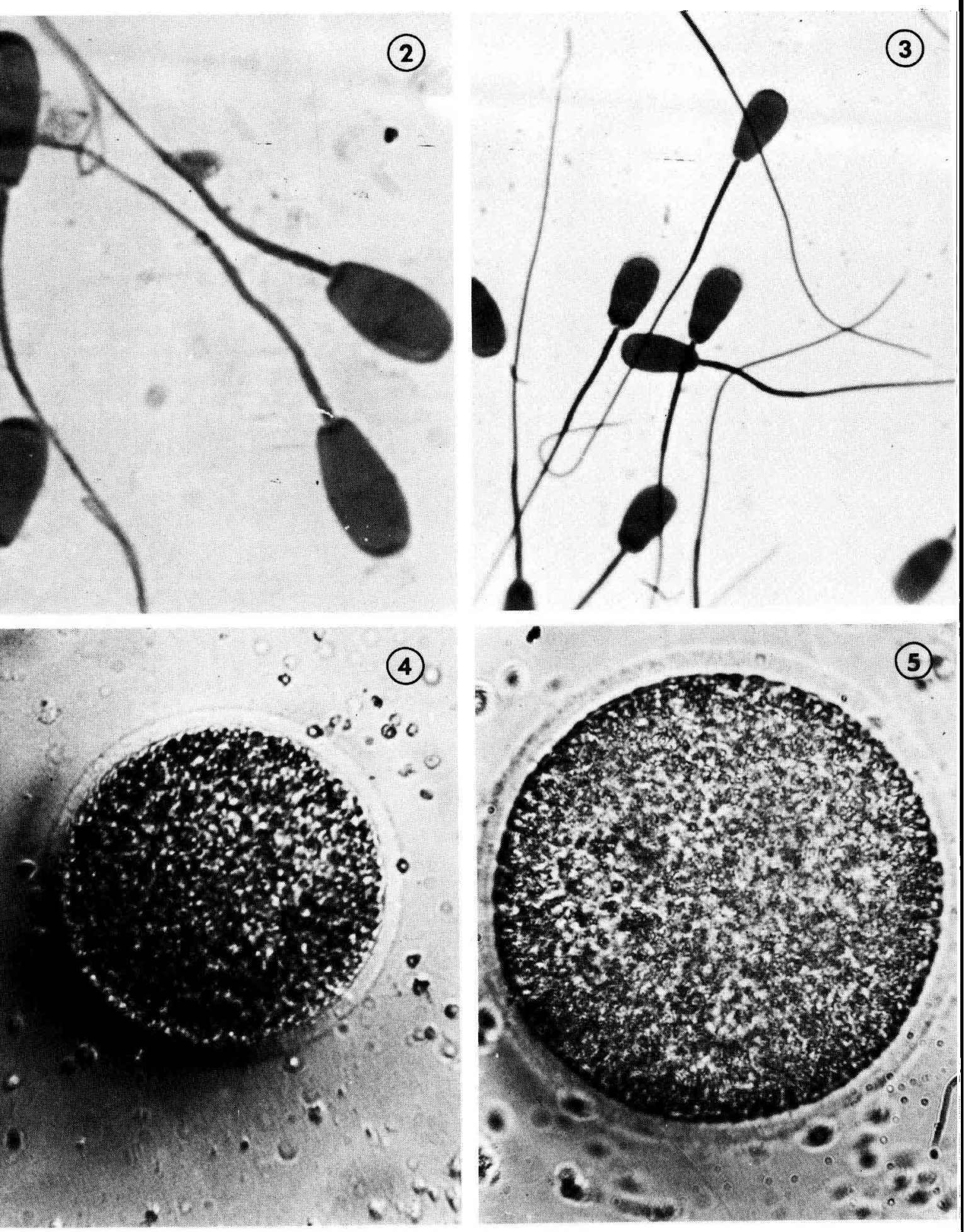

$3^{4} \cdot$

(5)

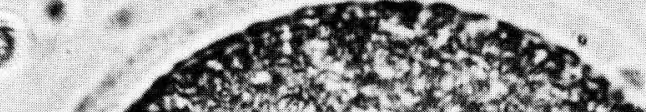
El

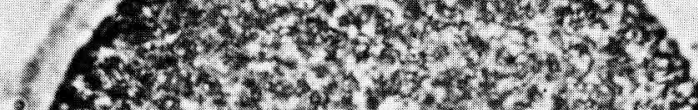

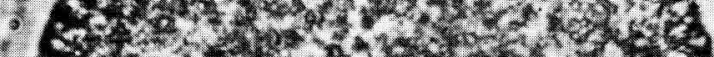

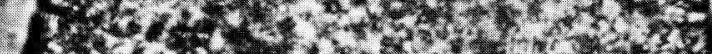

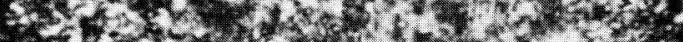

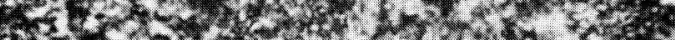

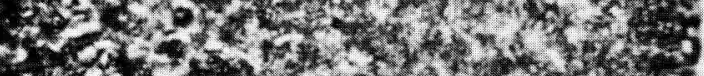

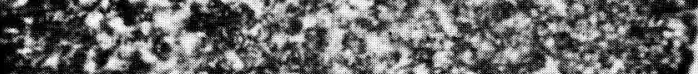

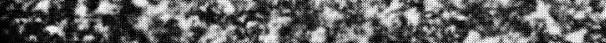

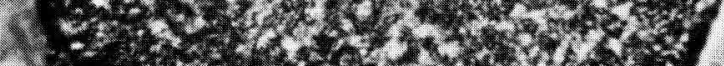

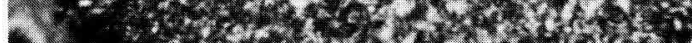

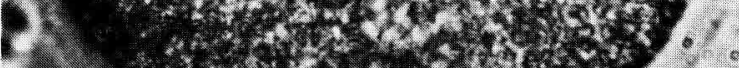

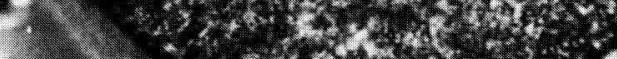

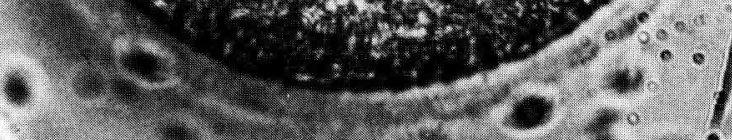

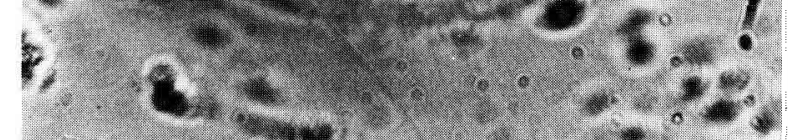




\section{Conclusion.}

Nous croyons que les résultats acfuels, bien qu'ils soient négatifs pour le sperme, nous incitent à poursuivre les essais. En effet, contrairement à ce qu'on aurait dû attendre de pressions aussi élevées, les cellules sont restées intactes et nous croyons qu'il existe une zone de pression qui n'entraîne pas de désordre dans les structures. Si cette hypothèse est exacte, on pourrait définir un moyen de conserver le sperme, éventuellement des embryons et toute autre cellule, sans recourir à des protecteurs de congélation.

Reçu en mai 1978

Accepté en octobre 1978.

Remerciements. - Nous tenons à remercier ici les Communautés Economiques Européennes et la Députation Permanente de la Province du Brabant qui nous ont permis de réaliser ce travail.

\section{Références}

BASSET J., MACHEBOEUF A., 1932. Ełude sur les effets biologiques des ultra-pressions. Résistance des bactéries des diastases et des toxines aux pressions très élevées. C. R. Acad. Sci., 195, 1431-1433.

BASSET J., MACHEBOEUF A., 19330. Etude sur les effets biologiques des ultra-pressions. Etudes sur l'immunité. Influence des pressions très élevées sur certains antigènes et anticorps. $C$. $R$. Acad. Sci., 196, 67-69.

BASSET J., MACHEBOEUF A., 1933b. Note résumant les résultats obtenus dans l'étude de l'influence des très hautes pressions sur les micro-organismes ef les ferments. Ergebn. Enzymforsch. Leipzig, 1, 304.

BASSET J., MACHEBOEUF A., WOLLMAN F., 1937. Ełudes biologiques effectuées grâce aux ultrapressions. Recherches sur les microbes pathogènes ef leurs toxines et sur les virus invisibles, les bactériophages et les tumeurs malignes. Ann. Inst. Pasteur, 58, 58-77.

BENTHAUS J., 1937. The effect of high pressure on tissue culture. Arch. ges. Physiol., 239, 107.

BORDET P., CHOME N., 1950. Propriétés du sérum soumis à l'action des hautes pressions. Ann. Inst. Pasteur, 79, 532-547.

BRIDGEMAN P. W., 1914. The coagulation of albumen by pressure. J. biol. Chem., 19, 511-512.

CATTELL M., 1936. The physiological effects of pressure. Biol. Rev., 11, 441.

DEUTICKE H. J., HARREN F., 1938. The course of enzymic reactions at high pressures. $Z$. Physiol. Chem., 256, 169.

DEVUYST A., HENRIET L., 1960. Le blocage du métabolisme du spermatozoïde par voie chimique. $25^{\circ}$ Anniv. Fond. Ist. ital. « Lazarro Spallanzani ».

DOW R. B., MATTHEWS I. D., 1939. The desintegration of erythrocytes and denaturation of hemoglobin by high pressure. Philos. Mag., 7, XXVII, 637.

DOW R. B., MATTHEWS J. E., THORP W. T. S., 1940. The effect of high pressure treatment on the physiological activity of insulin. Am. J. Physiol., 131, 382.

GRUNDFEST H., 1936. Effects of hydrostatic pressure upon the excitability, the recovery and the potential sequence of frog nerve. Cold Spr. Harb. Symp. quant. Biol., 4, 197.

HENRIET L., 1964. Rapport du Centre expér. Insémin. arfif. Univ. de Louvain. 34-36.

HILDEBRAND C. E., POLLARD E. C., 1972. Hydrostatic pressure effects on protein synthesis. Biophys. J., 12, 1235-1249.

JOHNSON F. H., CAMPBELL D. H., 1945. The retardation of protein denaturation by hydrostatic pressure. J. cell. comp. Physiol., 26, 43-46.

LAUFFER M. A., DOW R. B., 1941. The denaturation of tobacco mosaic virus at high pressures. J. biol. Chem., 140, 509-518. 
NAKASIMA K., IKEDA M., 1941. Hydrolisis of proteins at high temperatures and pressures. J. agr. Chem. Soc. Japan, 17, 295.

PEASE D. C., REGNERY D., 1941. Drosophila salivary chromosomes subjected to high hydrostatic pressures. J. cell. comp. Physiol., 17, 397.

POLGE C., SMITH A. U., PARKES A. S., 1949. Revival of spermatozoa after vitrification and dehydration at low temperature. Nature, London, 164, 666.

STEFANELLI A., 1973. L'effetto biologica delle elevate pressioni idrostatiche. Realizzazione di alcuni apparecchi per l'osservazione deretta del materiale. Lincei-Redn. Sci. Fis. Mat. Nat., 52, 569-572.

STEFANELLI A., ZACCHEI A. M., 1972. Prime osservazioni sperimentali sui progensi morfogenetici di embrioni di Anuri sottoposti ad elevate pressioni idrostatiche. Lincei-Rend. Sci. Fis. Mat. Nat., 52, 573-577.

THOMANEK U. F., FARAK F., MOSSBAUER R. L., FORMANEK H., SCHWAGER P., HOPPE W., 1973. Freezing of myoglobin crystals at high pressure. Acta Cryst., A 29, 263-265. 